\title{
Étude des possibilités de la méthode X-FEM pour la simulation numérique de la coupe
}

\author{
Yosra Guétari ${ }^{a}$, Steven Le Corre et Nicolas MoËs \\ Institut de Recherche en Génie Civil et Mécanique, GeM- UMR CNRS 6183, École Centrale de Nantes, BP 92101-1, \\ Rue de la Noë, 44321 Nantes Cedex 03, France
}

Reçu le 25 février 2005, accepté le 21 mars 2005

\begin{abstract}
Résumé - Nous introduisons dans cet article une application de la méthode des éléments finis étendus (X-FEM) au cas de la simulation numérique de la coupe, qui se trouve encore confrontée à des limitations d'ordre numérique. La gestion du problème du contact à l'interface outil/copeau est présentée, et l'intérêt du couplage entre X-FEM et la méthode des level-sets pour ce problème est mis en valeur. Les résultats de la validation, effectuée sur des exemples typiques, sont détaillés. Les premières validations sur un test d'usinage sont ensuite présentées, pour une loi de comportement de type fluide newtonien. Nous dégageons enfin les améliorations futures que la méthode est à même d'apporter à la simulation numérique de la coupe.
\end{abstract}

Mots clés : Élements finis étendus (X-FEM) / usinage / contact / Level-Set

\begin{abstract}
Study of the X-FEM method possibilities for the simulation of machining. This paper introduces an application of the eXtended Finite Element Method (X-FEM) to the case of machining simulation, which is still subjected to numerical limitations. The treatment of the contact problem at the tool/chip interface is presented, and highlights the interest of the coupling of X-FEM with the Level Set method. Validation results are detailed through typical examples. We then present the first simulations of chip formation, using a newtonian fluid law for the machined material, and extract the future improvements that the method is able to provide to the cutting numerical simulation.
\end{abstract}

Key words: EXtended Finite Element Method (X-FEM) / machining / contact / Level-Set

\section{Introduction}

La mise en forme par enlèvement de la matière, l'usinage en particulier, est un procédé complexe qui fait depuis longtemps l'objet de nombreuses recherches. Les travaux initiaux de Merchant [1] ont entamé ces recherches par des modélisations mécaniques purement analytiques, qui ont ouvert la voie à des études plus poussées qui présentent toutes un but commun : quantifier l'influence des paramètres process (dont notamment la nature et la géométrie de la pièce et de l'outil) sur les sollicitations qui apparaissent pendant l'opération de coupe (déformations, taux de déformation, efforts, échauffements, frottements).

Ces modélisations analytiques ont rapidement révélé leurs limites face aux forts couplages qui sont mis en jeu lors de la formation du copeau. Les approches numériques

a Auteur correspondant : yosra.guetari@ec-nantes.fr se sont donc avérées nécessaires. Ces approches peuvent être divisées en trois catégories principales :

a) La méthode classique des éléments finis lagrangiens. Cette méthode présente deux avantages majeurs : elle permet une bonne représentation des lois de comportement et des variables internes, et offre une grande facilité et une excellente précision pour la description des surfaces libres. Pour le cas de l'usinage, ces méthodes lagrangiennes présentent toutefois l'inconvénient de conduire à d'importantes distorsions du maillage. Celles-ci impliquent l'emploi de méthodes ALE et de techniques de remaillage qui sont connues pour être coûteuses en temps et en ressources. Ceci limite les possibilités de simulations sur de longues distances. Un second inconvénient de ces méthodes est la difficulté de leur utilisation pour traiter les problèmes des localisations des déformations, particulièrement important dans le cas de la simulation de l'usinage. 
b) Les méthodes non classiques d'éléments finis lagrangiens, comme les méthodes meshless $[2,3]$ ou NEM [4]. Ces méthodes robustes permettent de résoudre la plupart des difficultés liées au maillage, mais des opérations plutôt triviales, comme la description des surfaces ou la prescription des conditions aux limites sont encore très délicates à traiter dans le cadre de ce formalisme.

c) Les approches eulériennes. Dans ces approches, le problème de la grille maillée est le fait que le maillage ne coïncide pas avec le bord du corps en cours de déformation. Nous remédions à cette difficulté en utilisant la méthode X-FEM [5] qui permet aux éléments de contenir une partie de vide [6]. La position de la surface sur la grille eulérienne est repérée par une level-set $[7,8]$. Le remaillage n'est donc plus nécessaire. Une autre qualité de la méthode X-FEM, qu'elle doit au couplage avec la technique Level-Set, est la simplicité qu'elle offre pour le traitement du contact, comme nous le montrerons plus loin.

Malgré leurs avantages, ces techniques manifestent toutes des limites communes face au problème de simulation de l'usinage des métaux. Ces limites sont surtout liées aux critères de rupture du maillage, et au sens physique de quelques instructions actuellement utilisées (à l'instar de kill element dans les méthodes lagrangiennes). En effet, sans critère additionnel, il serait par exemple impossible de simuler la fragmentation des copeaux. Des méthodes comme l'introduction de l'endommagement aident à franchir ce cap, mais manquent encore de justifications expérimentales. De plus, le traitement de la localisation de la déformation, point bloquant et rarement abordé, nécessite une technique numérique supplémentaire.

Dans le cadre de nos travaux, nous présentons ici les premiers développements vers une approche originale de la simulation numérique de la coupe, qui porte en particulier sur l'implémentation d'un algorithme de contact outil/matière dans le contexte X-FEM/Level-Set. Nous en dégagerons les avantages, et nous discuterons les perspectives de ces travaux qui visent à surpasser - au moins en partie - les principales difficultés auxquelles les techniques actuelles de simulation numérique de la coupe sont confrontées.

\section{Développements}

\subsection{Problème global}

Nous abordons le traitement du problème par une formulation incompressible mixte en vitesse - pression $(v-p)$ écrite dans un contexte non-linéaire. La figure 1 décrit la géométrie du problème global : $(D)$ est le domaine maillé de fond, $(\Omega)$ est le volume d'un corps décrit par une fonction level-set, et mobile dans $(D)$ avec une vitesse donnée $\boldsymbol{v}_{\mathrm{d}}$, imposée sur une partie $\left(\Gamma_{\mathrm{d}}\right)$ de son bord $\left(\Gamma_{v}\right)$, bord où les vitesses sont imposées. Une condition de symétrie est imposée sur la partie inférieure $\left(\Gamma_{\mathrm{s}}\right)$ de $\left(\Gamma_{v}\right)$, et le reste du bord de $(\Omega)$ est libre.

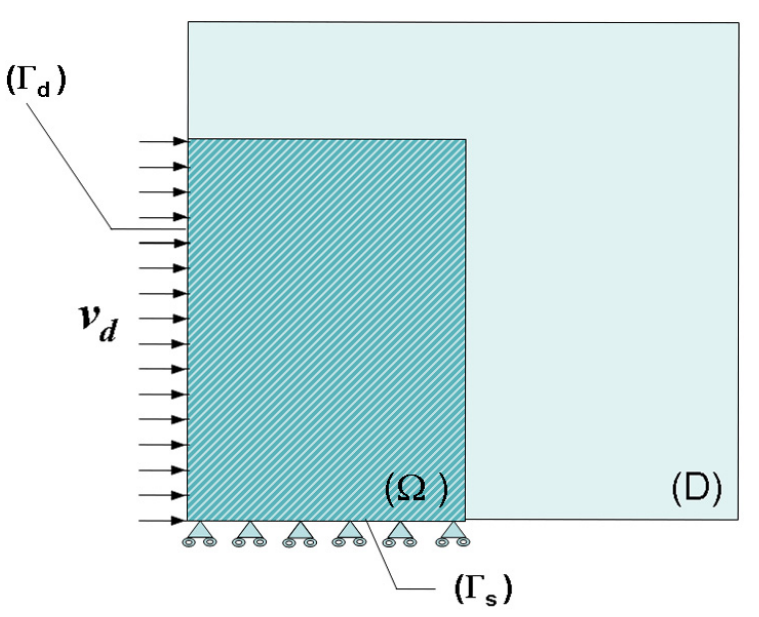

Fig. 1. Schéma du problème global.

Le système $(S)$ exprime la formulation variationnelle pour ce problème en termes de $v$ et $p$ :

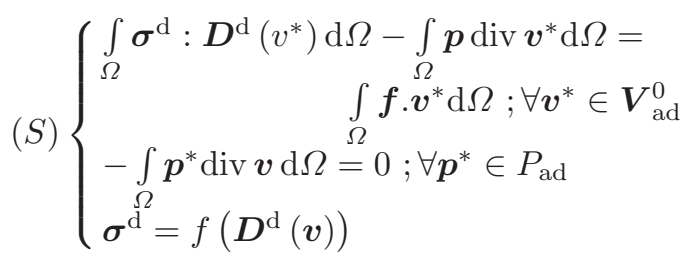

où $\boldsymbol{\sigma}^{\mathrm{d}}$ est la partie déviatoire du tenseur des contraintes de Cauchy, $\boldsymbol{D}^{\mathrm{d}}$ est la partie déviatoire du tenseur des taux de déformation, et $\boldsymbol{V}_{\text {ad }}^{0}$ et $P_{\text {ad }}$ sont définis par :

$$
\begin{aligned}
& \boldsymbol{V}_{\text {ad }}^{0}=\left\{\boldsymbol{v} \mid \boldsymbol{v} \in\left(H^{1}(\Omega)\right)^{n}, \boldsymbol{v}=0 \operatorname{sur}\left(\Gamma_{v}\right)\right\} \\
& P_{\mathrm{ad}}=\{p \mid p \text { est } \text { « régulier } »\}
\end{aligned}
$$

La condition de stabilité de Babuška-Brezzi est satisfaite par l'utilisation d'un élément quadratique en vitesse et linéaire en pression (interpolation P2/P1). La stabilité de cet élément dans le contexte X-FEM a été étudiée dans [9].

\subsection{Le problème du contact}

En vue d'effectuer une simulation de l'opération de coupe par éléments finis, un algorithme de contact doit être implémenté pour traiter l'interaction mécanique entre la pièce mobile et l'outil coupant. Cette interaction est introduite dans $(S)$ par le biais d'un terme surfacique supplémentaire :

$$
\left(\boldsymbol{S}^{\prime}\right)\left\{\begin{array}{l}
\int_{\Omega} \boldsymbol{\sigma}^{\mathrm{d}}: \boldsymbol{D}^{\mathrm{d}}\left(\boldsymbol{v}^{*}\right) \mathrm{d} \Omega-\int_{\Omega} \boldsymbol{p} \operatorname{div} \boldsymbol{v}^{*} \mathrm{~d} \Omega= \\
\quad \int_{\Omega} \boldsymbol{f} \cdot \boldsymbol{v}^{*} \mathrm{~d} \Omega+\int_{\Gamma_{\mathrm{c}}} \boldsymbol{T}(\boldsymbol{v}) \cdot \boldsymbol{v}^{*} \mathrm{~d} S ; \forall \boldsymbol{v}^{*} \in \boldsymbol{V}_{\mathrm{ad}}^{0} \\
-\int_{\Omega} \boldsymbol{p}^{*} \operatorname{div} \boldsymbol{v} \mathrm{d} \Omega=0 ; \forall p^{*} \in \boldsymbol{P}_{\mathrm{ad}} \\
\boldsymbol{\sigma}^{\mathrm{d}}=f\left(\boldsymbol{D}^{\mathrm{d}}(\boldsymbol{v})\right)
\end{array}\right.
$$



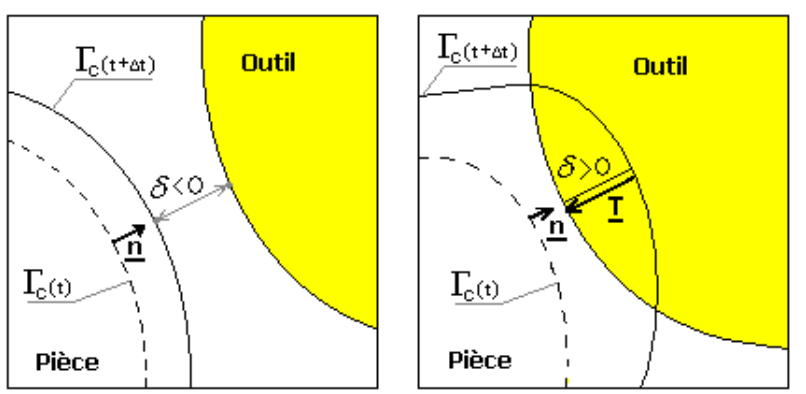

Fig. 2. Principe de la méthode de pénalisation du contact.

Dans $\left(S^{\prime}\right), \boldsymbol{T}$ est la contrainte de contact et $\left(\Gamma_{\mathrm{c}}\right)$ est le bord de la pièce où un contact est susceptible d'avoir lieu (ce bord est décrit par l'isovaleur zéro de la fonction levelset décrivant la pièce). Nous adoptons ici une méthode de pénalisation. Dans un tel contexte, $T$ est donnée par la relation (3):

$$
\boldsymbol{T}=-\chi \delta \boldsymbol{n}
$$

où $\chi$ est un coefficient de pénalisation arbitraire, dont la valeur doit être soigneusement choisie. $\boldsymbol{n}$ est la normale unitaire sortante de la pièce, évaluée dans la configuration du pas de temps précédent, et $\delta$ est la profondeur algébrique de la pénétration de la pièce dans l'outil. La valeur de $\delta$ est positive lorsqu'une pénétration numérique a effectivement eu lieu (Fig. 2). Il s'agit donc d'une formulation unilatérale du contact où les effets de frottement ne sont pas pris en compte pour le moment. L'effort $\boldsymbol{T}$ y est équivalent à une force élastique par unité de surface, proportionnelle à la pénétration $\delta$.

Dans le contexte X-FEM, la méthode de pénalisation est basée sur la technique Level Set. L'évaluation de $\delta$ est effectuée en évaluant la level-set associée à l'outil aux points de Gauss du bord de la pièce (Éq. (4)). Ce couplage offre un avantage numérique certain : la recherche des éléments de la surface en vis-à-vis n'est plus nécessaire, et il en va de même pour le calcul des normales aux surfaces en contact. Celles-ci sont directement données par la dérivation des fonctions level-sets correspondantes (Éq. (5)). Dans la relation (4), en nous basant sur le fait que les frontières de la pièce et de l'outil sont très rapprochées lorsqu'un contact a effectivement lieu, nous approximons la normale $\boldsymbol{n}$ par le vecteur opposé à la normale unitaire sortante de l'outil $\left(\boldsymbol{n}_{\text {outil }}\right): \boldsymbol{n}_{\text {outil }} \approx-\boldsymbol{n}$. Cette approche est par ailleurs pressentie pour simplifier l'intégration de modèles de frottement et éviter des aberrations numériques

$$
\begin{aligned}
& \delta\left(\boldsymbol{x}^{\boldsymbol{t}+\Delta t}\right)=-l s_{\text {outil }}\left(\boldsymbol{x}^{\boldsymbol{t}}+\boldsymbol{v}^{\boldsymbol{t}+\Delta t} \Delta t\right) \\
& \boldsymbol{n}_{\text {tool }}=\nabla l s_{\text {outil }}\left(\boldsymbol{x}^{\boldsymbol{t}}+\boldsymbol{v}^{\boldsymbol{t}+\Delta \boldsymbol{t}} \Delta t\right)
\end{aligned}
$$

Un schéma itératif de Newton-Raphson est utilisé pour linéariser le problème $\left(S^{\prime}\right)$. L'algorithme de contact boucle sur les points de la frontière de la pièce et itère la résolution jusqu'à ce que toutes les valeurs des pénétrations soient négatives $(\leq 0)$. Le mouvement de la pièce, effectué par un transport du domaine actuel par

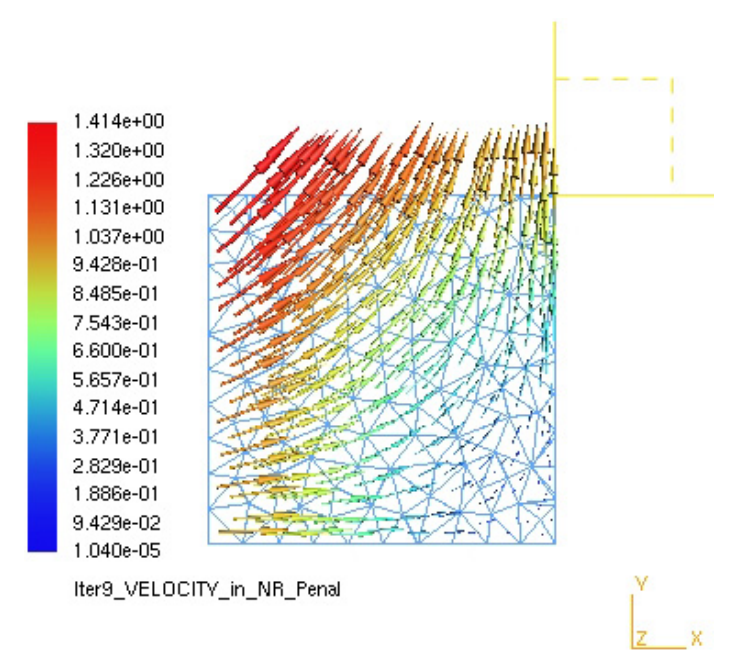

Fig. 3. Test homogène - Champs de vitesses.

propagation de la level-set de la pièce, est alors possible dès convergence du schéma de Newton-Raphson. Le transport de la level-set de la pièce est pris en charge par un algorithme séparé qui opère en trois temps :

- Extension du champ de vitesse connu du bord de la pièce (iso-zéro de la level-set) au domaine entier ;

- Propagation de la level-set selon ce champ de vitesse;

- Réinitialisation de la level-set en vue de retrouver une fonction distance signée qui prend en compte le mouvement effectué.

\section{Tests de validation et résultats}

\subsection{Test homogène}

Le premier test de validation (problème de Signorini) consiste à mettre en contact un corps mobile et obstacle fixe rigide. Il est équivalent à un essai de compression simple en déformations planes. D'excellentes solutions sont obtenues même avec l'utilisation de maillages grossiers.

\subsection{Le squeeze-test}

Le matériau mobile est ici une structure élancée $(h \ll$ $L)$ subissant une compression cisaillante contre un obstacle rigide fixe (Fig. 4). $v_{y}$ est fixée à zéro sur $\left(\Gamma_{\mathrm{d}}\right)$. La validation est effectuée sur un pas de temps, en démarrant d'une situation de quasi-contact. La figure 5a montre le résultat en terme de vitesse. Lorsque le contact est établi, nous vérifions que la solution en vitesse selon $y$ est parabolique sur une section horizontale du corps mobile (Fig. 5b).

La solution analytique en termes de pression est donnée par la solution de Hele-Shaw, issue de modèles d'injection-compression de polymères. Si $y$ est la coordonnée dans la direction verticale, alors :

$$
P_{\mathrm{a}}=12 \mu V_{\mathrm{d}}\left(L^{2}-y^{2}\right) / h^{3}
$$




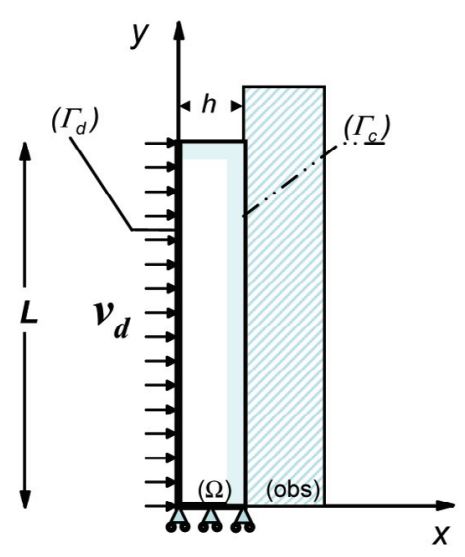

Fig. 4. Le squeeze-test.

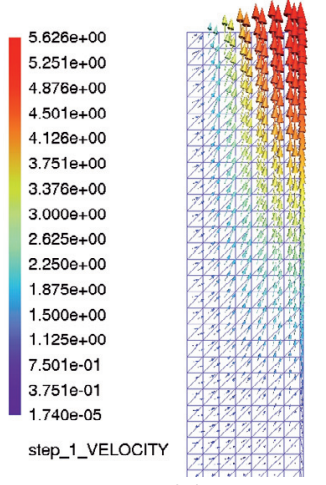

(a)

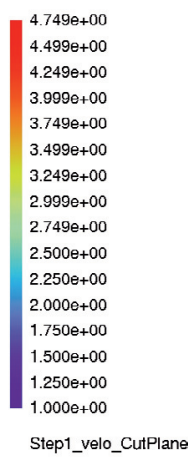

Step1_velo_CutPlane
Fig. 5. (a) Champ de vitesse pour le cas du squeeze-test. (b) Vitesse sur une section du corps mobile.

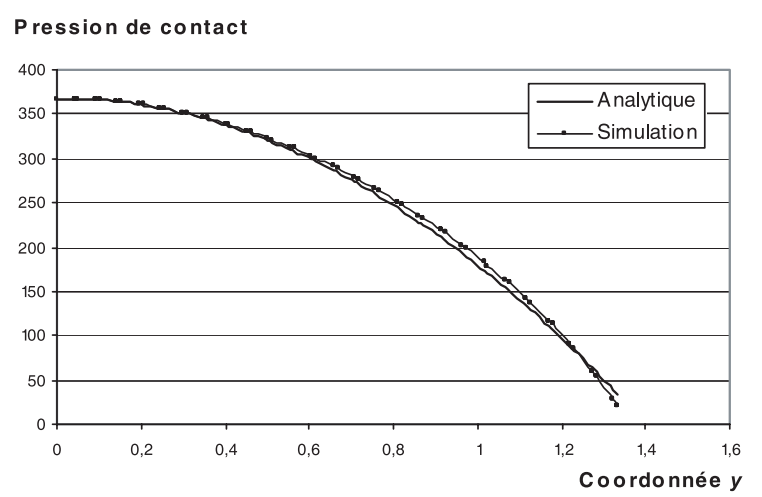

Fig. 6. Squeeze test : comparaisons des pressions analytique et numérique.

Nous vérifions la bonne concordance des solutions analytique et expérimentale sur le bord $\left(\Gamma_{\mathrm{c}}\right)$ du corps mobile (Fig. 6).

\section{3 Écrasement d'une goutte}

Le test d'une goutte (en 2D) entrant en contact avec une surface plane devient simple à résoudre une fois les calculs précédents validés. Il s'agit d'un premier test incluant à la fois l'algorithme de propagation des level-sets

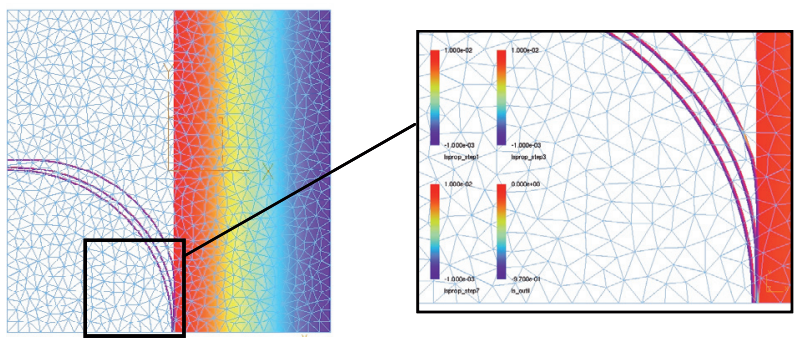

Fig. 7. Déformation du bord de la goutte sur la surface de contact.

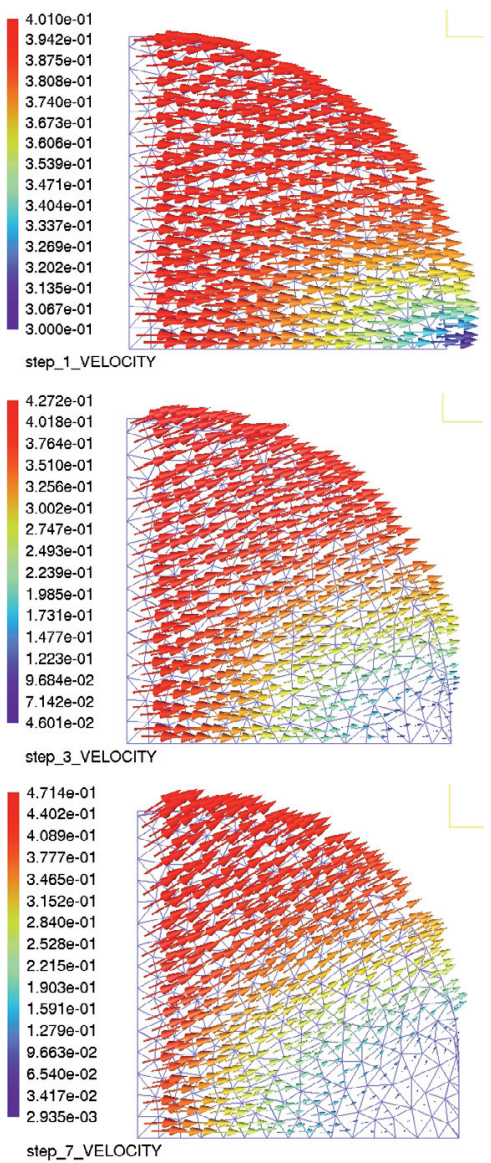

Fig. 8. Champ de vitesse pour l'écrasement d'une goutte (de haut en bas : temps $0,01,0,03$ et $\left.0,07 \mathrm{~s}-v_{\text {imp }}=0,4 \mathrm{~m} \cdot \mathrm{s}^{-1}\right)$.

et le traitement du contact. On notera que la solution est fortement conditionnée par un bon ajustement du coefficient de pénalisation. Moyennant cet ajustement, des profils de contact parfaitement réalistes sont obtenus pour ce problème (Figs. 7-8).

\subsection{Test d'usinage}

L'opération d'usinage met en jeu une pièce mobile et un outil fixe (Fig. 9a). À ce stade, nous procédons à une modélisation simplifiée de l'opération de coupe : l'outil est modélisé par un corps rigide fixe, et la pièce par un fluide newtonien de viscosité arbitraire (1 Pa.s). La pièce avance 


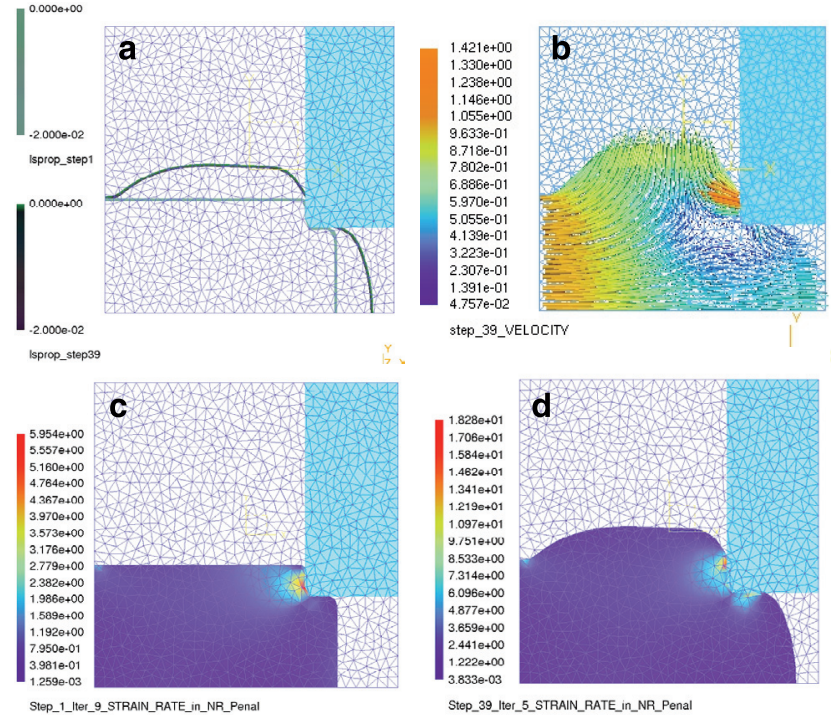

Fig. 9. (a) Déformation du matériau usiné ( $t=0,01$ et $0,39 \mathrm{~s})$. (b) Champ de vitesse $(t=0,39 \mathrm{~s})$ pour l'usinage du fluide newtonien. (c, d) Évolution du taux de déformation $(t=0,01$ et $0,39 \mathrm{~s})$.

vers l'outil avec une vitesse imposée (vitesse de coupe). La pièce et l'outil sont initialement en contact. Des résultats satisfaisants sont obtenus pour cet écoulement newtonien, mais notons qu'un modèle rhéologique aussi simpliste ne permet pas de traduire le comportement réel du matériau usiné (i.e. un comportement similaire à celui de la pièce, le plus souvent modélisé par des lois telles que le modèle de plasticité de Johnson-Cook, comme dans [10,11] par exemple). Toutefois, nous pouvons observer que le cisaillement produit l'augmentation attendue du taux de déformation et de la pression au voisinage du bec de l'outil ; mais aucune localisation n'a pu être mise en évidence dans la bande où la direction du champ de vitesse accuse un changement suite aux effets de contact (Fig. 9).

À ce stade et avec le comportement modélisé, seules des validations qualitatives peuvent être effectuées pour le cas de l'opération d'usinage. Des améliorations très sensibles peuvent à présent être apportées par l'utilisation de ce modèle de calcul, en y appliquant une loi de comportement plus adaptée.

\section{Conclusion}

Nous avons présenté une implémentation originale du traitement du contact exploitant les avantages offerts par le couplage entre X-FEM et la méthode Level-Set. Cette approche a permis de simplifier considérablement le principe de la simulation de l'enlèvement de copeau. Ceci constitue jusqu'ici l'avantage majeur obtenu pour cet axe de recherche. Des améliorations doivent toutefois être apportées pour dépasser quelques inconvénients de l'usage de X-FEM qui sont ceux des méthodes eulériennes en général : ces inconvénients sont liés à l'utilisation de lois de comportement pour les métaux, qui impliquent le transport de variables internes. Par ailleurs, le couplage de X-FEM avec l'enrichissement de l'approximation éléments finis permettra d'appréhender la localisation de la déformation à partir de la loi de comportement du matériau usiné, sans perdre la qualité de la solution éléments finis. Ceci est sans doute la perspective la plus prometteuse des développements futurs.

\section{Références}

[1] M.E. Merchant, Mechanics of the metal cutting process. I. Orthogonal cutting and a type 2 chip, J. Appl. Phys. 16 (1945) 267-275. Mechanics of the metal cutting process. II. Plasticity conditions in orthogonal cutting, J. Appl. Phys. 16 (1945) 318-324

[2] T. Belytschko, Y. Lu, L. Gu, Element free Galerkin methods, Int. J. Num. Meth. Eng. 37 (1994) 229-256

[3] B. Nayroles, G. Touzot, P. Villon, Generalizing the finite element method: Diffuse approximation and diffuse elements, Comp. and Struct. 10 (1992) 307-318

[4] M.A. Martinez, E. Cueto, M. Doblaré, F. Chinesta, Natural element meshless simulation of flows involving short fiber suspensions, J. Non-Newt., Fluid Mech. 115 (2003) 51-78

[5] J. Dolbow, N. Moës, T. Belytschko, An extended finite element method for modeling crack growth with frictional contact, Comp. Meth. Appl. Mech. Eng. 190 (2001) $5825-6846$

[6] C. Daux, N. Moës, J. Dolbow, N. Sukumar, T. Belytschko, Arbitrary branched and intersecting cracks with the eXtended Finite Element Method, Int. J. Num. Meth. Eng. 48 (2000) 1741-1760

[7] J.A. Sethian, Level Set methods and fast marching methods: Evolving interface in computational geometry, fluid mechanics, computer vision, and mechanical science, Cambridge university press, Cambridge, UK, 1999

[8] N. Sukumar, D.L. Chopp, N. Moës, T. Belytschko, Modeling holes and inclusions by Level-Sets in the eXtended Finite Element Method, Comp. Meth. Appl. Mech. Eng. 190 (2001) 6183-6200

[9] G. Legrain, N. Moës, A. Huerta, Stability of incompressible formulation enriched with X-FEM, Comp. Meth. Appl. Mech. Eng., submitted

[10] B. Lesourd, Étude et modélisation des mécanismes de formation de bandes de cisaillement intense en coupe des métaux - Application au tournage assisté LASER de l'alliage de titane TA6V, Thèse, École centrale de Nantes, France, 1996

[11] B. Changeux, Loi de comportement pour l'usinage Localisation de la déformation et aspects microstructuraux, Thèse, ENSAM de Paris, France, 2001 